Effie Papageorgiou*

\title{
Riesz means on homogeneous trees
}

https://doi.org/10.1515/conop-2020-0111

Received August 18, 2020; accepted January 26, 2021

Abstract: Let $\mathbb{T}$ be a homogeneous tree. We prove that if $f \in L^{p}(\mathbb{T}), 1 \leq p \leq 2$, then the Riesz means $S_{R}^{z}(f)$ converge to $f$ everywhere as $R \rightarrow \infty$, whenever $\operatorname{Re} z>0$.

Keywords: Homogeneous trees, Riesz means

MSC: 43A90, 22E30

A homogeneous tree $\mathbb{T}$ of degree $Q+1, Q \geq 2$, is an infinite connected graph with no loops, in which every vertex is adjacent to $Q+1$ other vertices. We shall identify $\mathbb{T}$ with its set of vertices $\mathcal{V}$. $\mathbb{T}$ carries a natural distance $d$ and a natural measure $\mu$. Specifically, $d(x, y)$ is the number of edges of the shortest path joining $x$ to $y$ and $\mu$ is the counting measure. For the counting measure, the volume of any sphere $S(x, n)$ in $\mathbb{T}$ is given by

$$
|S(x, n)|= \begin{cases}1, & \text { if } n=0 \\ (Q+1) Q^{n-1}, & \text { if } n \in \mathbb{N},\end{cases}
$$

and the Lebesgue spaces, associated with $\mu$, have norms defined by $L^{p}(\mathbb{T})$

$$
\|f\|_{p}= \begin{cases}\left(\int_{\mathbb{T}}|f(x)|^{p} d \mu\right)^{1 / p}=\left(\sum_{x \in \mathbb{T}}|f(x)|^{p}\right)^{1 / p}, & \text { if } 1 \leq p<\infty \\ \sup _{x \in \mathbb{T}}|f(x)|, & \text { if } p=\infty .\end{cases}
$$

Let us fix a base point $x_{0}$ and set $|x|=d\left(x, x_{0}\right)$. Functions depending only on $|x|$ are called radial. If $E(\mathbb{T})$ is a function space on $\mathbb{T}$, we will denote by $E(\mathbb{T})^{\#}$ the subspace of radial elements in $E(\mathbb{T})$.

Let $G$ be the group of isometries of $\mathbb{T}$, and suppose that $G$ acts transitively on $\mathbb{T}$. If $x_{0} \in \mathbb{T}$ is a fixed vertex, then the orbit $G x_{0}$ is all of $\mathbb{T}$. Therefore $\mathbb{T}$ may be identified through the map $g \mapsto g x_{0}$ with the quotient $G / K$, where $K=\left\{g \in G: g x_{0}=x_{0}\right\}$, [9, p. 46]. This means that every function on $\mathbb{T}$ may be lifted to a function on $G$, by defining $\tilde{f}(g)=f\left(g x_{0}\right)$. The function $\tilde{f}$ has the property that $\tilde{f}(g k)=\tilde{f}(g)$, for every $k \in K$, and conversely a $K$-right-invariant function on $G$ may be identified with a function on $\mathbb{T}$. Thus, we shall identify functions defined on $\mathbb{T}$ with right- $K$-invariant functions on $G$ and radial functions with $K$-bi-invariant functions on $G$.

Let $G$ be a locally compact group and $K$ be a compact subgroup of $G$; then the pair $(G, K)$ is called a Gelfand pair if the space $C_{c}(K \backslash G / K)$ of complex continuous $K$-bi-invariant functions with compact support is a commutative algebra with the convolution product (the space $C_{c}(K \backslash G / K)$ is always an algebra with the convolution product). Under the hypothesis that $K$ acts transitively on the boundary of $\mathbb{T},(G, K)$ is a Gelfand pair. Also, the transitive action of $K$ on the boundary of $\mathbb{T}$ is also a necessary condition for $(G, K)$ to be a Gelfand pair, [9, p. 47]. In fact, the following holds true, [22].

Proposition. For every finite subset $\mathcal{F}$ of $\mathcal{V}$, denote by Aut $\mathcal{F}_{\mathcal{F}}(\mathbb{T})$ the group of automorphisms $g$ of $\mathbb{T}$ such that $g(x)=x$ for all $x \in \mathcal{F}$. Then $G$ is equipped with a topology of locally compact totally discontinuous group such that the subgroups Aut $\mathcal{F}_{\mathcal{F}}(\mathbb{T})$ form a fundamental system of neighborhoods of the identity in G. Moreover a subgroup $H$ is maximal, open, compact in $G$ if and only if $H$ is the stabiliser of a point $x$ in $V$.

^Corresponding Author: Effie Papageorgiou: Department of Mathematics, Aristotle University of Thessaloniki, Thessaloniki 54.124, Greece 
We normalize the Haar measure on $G$ in such a way that $K$ has a unit mass. Then

$$
\sum_{x \in \mathbb{T}} f(x)=\int_{G} f(g) d g, f \in L^{1}(\mathbb{T}) .
$$

This allows us to define the convolution of two functions on $\mathbb{T}$ by

$$
\left(f_{1} \star f_{2}\right)(g)=\int_{G} f_{1}(h) f_{2}\left(h^{-1} g\right) d h, g \in G .
$$

If $f_{2}$ is radial, then (2) rewrites

$$
\left(f_{1} \star f_{2}\right)(x)=\sum_{n \geq 0} f_{2}(n) \sum_{y \in S(x, n)} f_{1}(y), x, y \in \mathbb{T} .
$$

In this short note, we deal with the Riesz means $S_{R}^{z}, \operatorname{Re} z>0, R>0$, on $\mathbb{T}$, which are defined as convolution operators

$$
S_{R}^{z} f=f^{\star} \kappa_{R}^{z},
$$

where $\kappa_{R}^{z}$ is the inverse spherical transform of the multiplier

$$
m_{R}^{z}(\lambda)=\left(1-\frac{1-\gamma(\lambda)}{R}\right)_{+}^{z},
$$

(see Section 1 for more details). We prove the following result.

Theorem 1. Let $1 \leq p \leq 2$. If $\operatorname{Re} z>0$, then for $f \in L^{p}(\mathbb{T})$,

$$
\lim _{R \rightarrow+\infty} S_{R}^{z} f(x)=f(x), \text { everywhere. }
$$

Let us recall that the Riesz means were first treated by E.M.Stein in [19], where he proved that if $f \in$ $L^{p}\left([0,1]^{n}\right), n \geq 1, p \in(1,2]$, then

$$
\left\|S_{R}^{z}(f)-f\right\|_{p} \longrightarrow 0, \text { as } R \longrightarrow \infty
$$

whenever $\operatorname{Re} z>\left(\frac{n-1}{2}\right)\left(\frac{2}{p}-1\right)$. Since then, many authors have studied Riesz means in various geometric contexts, as euclidean spaces, compact manifolds, Lie groups of polynomial volume growth, graphs and discrete groups of polynomial volume growth, Riemannian manifolds of nonnegative curvature, compact semisimple Lie groups and noncompact symmetric spaces. See [1, 3, 4, 10-12, 14, 16, 18, 19, 21].

Note that the multiplier $m_{R}^{z}(\lambda)$ does not extend holomorphically to any strip containing the real line. So, by [7, Theorem 1.2], the Riesz means operator is not bounded on $L^{p}(\mathbb{T})$ if $p \neq 2$ and consequently the norm summability problem on $L^{p}(\mathbb{T}), p \neq 2$, is ill posed. Note also that in the case of homogeneous trees, there is no restriction on the size of $\operatorname{Re} z>0$, contrarily to the Euclidean case and even to the hyperbolic space case, due to absence of local obstructions.

\section{Preliminaries}

In this section we present the tools we need for the proof of our results. For details, see for example [7, 8]. Let $\mathcal{M}$ be the mean operator

$$
(\mathcal{M} f)(x)=\frac{1}{Q+1} \sum_{y \in \mathbb{T}, d(x, y)=1} f(y) .
$$

Then, the Laplacian $\mathcal{L}$ on $\mathbb{T}$ is defined by

$$
\mathcal{L}=I-\mathcal{M} .
$$


The spherical function $\varphi_{\lambda}$ of index $\lambda \in \mathbb{C}$ is the unique radial eigenfunction of the operator $\mathcal{L}$, which is associated with the eigenvalue

$$
\gamma(\lambda)=\frac{Q^{i \lambda}+Q^{-i \lambda}}{Q^{\frac{1}{2}}+Q^{-\frac{1}{2}}}=\frac{2}{Q^{\frac{1}{2}}+Q^{-\frac{1}{2}}} \cos (\lambda \log Q),
$$

and which is normalized by $\varphi_{\lambda}(0)=1$. Set $\tau=\frac{2 \pi}{\log Q}$. Then,

$$
\gamma(\lambda)=\gamma(0) \cos \left(\frac{2 \pi}{\tau} \lambda\right)
$$

Note that $\varphi_{\lambda}$ is periodic with period $\tau$.

The spherical Fourier transform of a radial function $f$ on $\mathbb{T}$ is defined by

$$
(\mathcal{H} f)(\lambda)=\sum_{x \in \mathbb{T}} f(x) \varphi_{\lambda}(x)=f(0)+\sum_{n \geq 1}(1+Q) Q^{n-1} f(n) \varphi_{\lambda}(n), \quad \lambda \in \mathbb{C} .
$$

The following inversion formula holds:

$$
\left(\mathcal{H}^{-1} f\right)(x)=\int_{-\tau / 2}^{\tau / 2} f(\lambda) \varphi_{\lambda}(x) \frac{d \lambda}{|\mathbf{c}(\lambda)|^{2}}, \quad x \in \mathbb{T},
$$

where $\mathbf{c}$ is the meromorphic function

$$
\mathbf{c}(z)=\frac{1}{Q^{1 / 2}+Q^{-1 / 2}} \frac{Q^{1 / 2+i z}-Q^{-1 / 2-i z}}{Q^{i z}-Q^{-i z}}, \quad z \in \mathbb{C} \backslash\left(\frac{\tau}{2}\right) \mathbb{Z} .
$$

We have the following Plancherel theorem [7]: the spherical Fourier transform extends to an isometry of $L^{2}(\mathbb{T})^{\#}$ onto $L^{2}\left([-\tau / 2, \tau / 2], \frac{d \lambda}{|\mathbf{c}(\lambda)|^{2}}\right)$ and

$$
\|f\|_{2}=\left(\int_{-\tau / 2}^{\tau / 2}|(\mathcal{H} f)(\lambda)|^{2} \frac{d \lambda}{|\mathbf{c}(\lambda)|^{2}}\right)^{1 / 2} .
$$

Note also that the spherical Fourier transform is written as a composition

$$
\mathcal{H}=\mathcal{F} \circ \mathcal{A}
$$

of the euclidean Fourier transform $\mathcal{F}$ and the Abel transform $\mathcal{A}$ [8]. Recall that the kernel $\kappa_{R}^{z}$ of the Riesz means operator is given by the inverse spherical transform of the multiplier $m_{R}^{z}$ :

$$
\kappa_{R}^{z}=(\mathcal{F} \circ \mathcal{A})^{-1}\left(m_{R}^{z}\right)=\left(\mathcal{A}^{-1} \circ \mathcal{F}^{-1}\right)\left(m_{R}^{z}\right) .
$$

For that, we shall make use of the following inversion formulas, [8]:

$$
\left(\mathcal{F}^{-1} f\right)(n)=\frac{1}{\tau} \int_{-\tau / 2}^{\tau / 2} f(\lambda) Q^{-i \lambda n} d \lambda=\frac{1}{\pi} \int_{0}^{\pi} f\left(\frac{\tau}{2 \pi} \lambda\right) \cos (\lambda n) d \lambda,
$$

and

$$
\left(\mathcal{A}^{-1} f\right)(n)=\sum_{k=0}^{\infty} Q^{-\frac{n}{2}-k}\{f(n+2 k)-f(n+2 k+2)\} .
$$

\section{Estimates of the kernel $\kappa_{R}^{z}$}

Lemma 2. If $\operatorname{Re} z>0$, then

$$
\left|\kappa_{R}^{z}(n)\right| \leq c Q^{-n / 2} .
$$


Proof. We have that $\kappa_{R}^{z}=\mathcal{H}^{-1}\left(m_{R}^{z}\right)=\left(\mathcal{A}^{-1} \circ \mathcal{F}^{-1}\right)\left(m_{R}^{z}\right)$. Then, using (13), (12) and (4), we obtain the following explicit expression of $\kappa_{R}^{z}$ :

$$
\begin{aligned}
\kappa_{R}^{z}(n) & =\frac{1}{\pi} \sum_{k=0}^{\infty} Q^{-\frac{n}{2}-k} \int_{0}^{\pi}\left(1-\frac{1-\gamma\left(\frac{\tau}{2 \pi} \lambda\right)}{R}\right)_{+}^{z} \sin \lambda \sin (\lambda(n+2 k+1)) d \lambda \\
& =\frac{1}{\pi} Q^{-\frac{n}{2}} \sum_{k=0}^{\infty} Q^{-k} \int_{0}^{\pi} \sin \lambda \sin (\lambda(n+2 k+1)) d \lambda \\
& \leq c Q^{-\frac{n}{2}} \sum_{k=0}^{\infty} Q^{-k} \leq c Q^{-\frac{n}{2}} .
\end{aligned}
$$

Remark. Note that the same kernel estimate holds for every operator $m_{R}(\mathcal{L})$ with a uniformly bounded multiplier $\left|m_{R}(\lambda)\right| \leq c$.

\section{Proof of Theorem 1}

For the proof of Theorem 1, we need to introduce the maximal function associated with Riesz means:

$$
S_{\star}^{Z}(f)(x)=\sup _{R>0}\left|S_{R}^{z}(f)(x)\right|, f \in L^{p}(\mathbb{T}), 1 \leq p \leq 2 .
$$

The proof will be given in steps.

Step 1: The maximal operator $S_{\star}^{z}(f)$ is bounded from $L^{p}(\mathbb{T})$ to $L^{r}(\mathbb{T}), 1 \leq p \leq 2$, and $r \geq p q /(2-p+p q-q)$, for every $q>2$.

First, proceeding as in [12], we have that

Lemma 3. If $f \in L^{2}(\mathbb{T})$ and $z \in \mathbb{C}$ with $\operatorname{Re} z>0$, then $\left\|S_{*}^{z} f\right\|_{2} \leq c(z)\|f\|_{2}$.

Proof. We include the proof for the sake of completeness. Let $h_{R}, R>0$, be the heat kernel on $\mathbb{T}$, i.e. let the heat semigroup be $H_{R} f:=e^{-R \mathcal{L}} f=f \star h_{R}, f \in L^{2}(\mathbb{T})$. Since by [7, Theorem 2.2], it holds $\left\|e^{-R \mathcal{L}}\right\|_{L^{2}(\mathbb{T}) \rightarrow L^{2}(\mathbb{T})}=$ $e^{-(1-\gamma(0)) R} \leq 1$, by [20, Chapter III, MAXIMAL THEOREM], the heat maximal operator $f \rightarrow H_{\star} f:=\sup _{R>0}\left|f^{\star} h_{R}\right|$ is bounded on $L^{2}(\mathbb{T})$. Thus, it suffices to prove the boundedness of $\left(S^{z}-H\right)_{\star}$. Using the Mellin transform for the functions $(1-t / R)_{+}^{z}$ and $e^{-t R}, R>0,[2]$, and the spectral theorem for $\mathcal{L}$, we have

$$
\left(S_{R}^{z}-H_{R}\right) f=\int_{\mathbb{R}} c(z, s) R^{-i s}(\mathcal{L})^{i s} f d s,
$$

where $|c(z, s)| \leq c(z)(1+|s|)^{-(\operatorname{Re} z+1)}$, [12], thus the integral above converges. Since $L^{2}(\mathbb{T})$ is a complete Banach lattice, from [6], we can write

$$
\left(S^{z}-H\right)_{\star} f=\sup _{R>0}\left|\left(S_{R}^{z}-H_{R}\right) f\right| \leq c(z) \int_{\mathbb{R}}(1+|s|)^{-(\operatorname{Re} z+1)}\left|\mathcal{L}^{i s} f\right| d s .
$$

Thus,

$$
\left\|\left(S^{z}-H\right)_{\star} f\right\|_{L^{2}(\mathbb{T})} \leq c(z)\|f\|_{L^{2}(\mathbb{T})},
$$

since we have $\left\|\mathcal{L}^{i s}\right\|_{L^{2}(\mathbb{T}) \rightarrow L^{2}(\mathbb{T})} \leq 1$ by the spectral theorem and the fact that $\mathcal{L}$ is self-adjoint on $L^{2}(\mathbb{T})[7$, p.4271].

Lemma 4. Let $q>2$. If $f \in L^{p}(\mathbb{T}), p \in\left[1, q^{\prime}\right]$ and $z \in \mathbb{C}$ with $\operatorname{Re} z>0$, then $\left\|S_{\star} f\right\|_{r} \leq c\|f\|_{p}$, for $r \in\left[q p^{\prime} /\left(p^{\prime}-\right.\right.$ $q), \infty]$. 
Proof. Since the kernel $\kappa_{R}^{z}$ is radial, we have by (3) and Lemma 2 that

$$
\begin{aligned}
\left|S_{R}^{z} f(x)\right| & =\left|\left(f^{\star} \kappa_{R}^{z}\right)(x)\right|=\left|\sum_{n \geq 0} \kappa_{R}^{z}(n) \sum_{y \in S(x, n)} f(y)\right| \\
& \leq c \sum_{n \geq 0} Q^{-n / 2} \sum_{y \in S(x, n)}|f(y)| \\
& =c\left(|f|^{\star} \kappa_{Q}\right)(x),
\end{aligned}
$$

where $\kappa_{Q}(n)=Q^{-n / 2}$. Thus,

$$
\left|S_{\star}^{z} f(x)\right|=\sup _{R>0}\left|\left(f^{\star} \kappa_{R}^{z}\right)(x)\right| \leq c\left(|f|^{\star} \kappa_{Q}\right)(x),
$$

where $\kappa_{Q}(n)=Q^{-n / 2}$. Since the kernel $\kappa_{Q}$ is radial, as in [8, p.787], we have

$$
\left\|\kappa_{Q}\right\|_{q}^{q}=\left|\kappa_{Q}(0)\right|^{q}+(Q+1) \sum_{n=1}^{\infty} Q^{n-1}\left|\kappa_{R}^{z}(n)\right|^{q} .
$$

Thus, for every $q>2$, we have

$$
\begin{aligned}
\left\|\kappa_{Q}\right\|_{q}^{q} & \leq c+c(Q+1) \sum_{n=1}^{\infty} Q^{n-1} Q^{-\frac{n}{2} q} \\
& \leq c+c \frac{Q+1}{Q} \sum_{n=1}^{\infty} Q^{-\frac{n}{2}(q-2)}<c .
\end{aligned}
$$

From (15) and (16), for fixed $q>2$ and $\operatorname{Re} z>0$, the operator $S_{\star}^{z}$ maps $L^{p}(\mathbb{T}), p \in\left[1, q^{\prime}\right]$, continuously to $L^{r}(\mathbb{T})$, for every $r \in\left[q p^{\prime} /\left(p^{\prime}-q\right), \infty\right]$.

From Lemmata 3 and 4 and Riesz-Thorin interpolation, we obtain that for every $r \geq p q /(2-p+p q-q)$, there is $c(z)>0$ such that for every $f \in L^{p}(\mathbb{T}), 1 \leq p \leq 2$,

$$
\left\|S_{\star}^{z}\right\|_{r} \leq c(z)\|f\|_{p} .
$$

Step 2: Theorem 1 holds for a dense subspace $D$ of $L^{p}(\mathbb{T}), 1 \leq p \leq 2$.

We shall modify the proof of [1, Theorem 4]. Set

$$
D=\left\{e^{-\frac{1}{t} \mathcal{L}} e^{-s \mathcal{L}} f ; f \in C_{0}^{\infty}(\mathbb{T}), t \geq 1,0<s \leq 1\right\},
$$

and recall that the heat operator is $L^{p}(\mathbb{T})$ bounded for every $1 \leq p \leq 2$, [7]. Note thus that $\left\|e^{-s \mathcal{L}} f-f\right\|_{p} \rightarrow 0$ as $s \rightarrow 0$ for all $f \in C_{0}^{\infty}(\mathbb{T})$ and $1 \leq p \leq 2$. Also, the multiplier

$$
e^{-\frac{1}{t}(1-\gamma(\lambda))} e^{-s(1-\gamma(\lambda))}-e^{-s(1-\gamma(\lambda))}
$$

yields an $L^{p}$ bounded convolution operator, for every $1 \leq p \leq 2$. It follows from the triangle inequality that

$$
\left\|e^{-\frac{1}{t} \mathcal{L}} e^{-s \mathcal{L}} f-f\right\|_{p} \rightarrow 0, \text { as } t \rightarrow \infty .
$$

Thus, the space $D$ is dense to all $L^{p}(\mathbb{T}), 1 \leq p \leq 2$.

Let us now fix some $g=e^{-\frac{1}{t} \mathcal{L}} e^{-S \mathcal{L}} f \in D$. Let us also consider a function $\psi \in C^{\infty}(\mathbb{R})$ such that

$$
\psi(\lambda)= \begin{cases}1, & \text { for }|\lambda| \leq 1 / 4 \\ 0, & \text { for }|\lambda| \geq 1 / 2,\end{cases}
$$

and put $\psi_{R}(\lambda)=\psi(\lambda / R), R>0$. Then, for $R$ large enough we have that

$$
m_{R}^{z}(\mathcal{L}) g=\psi_{R}(\mathcal{L}) m_{R}^{z}(\mathcal{L}) e^{-\frac{1}{t} \mathcal{L}} e^{-s \mathcal{L}} f
$$


and therefore

$$
m_{R}^{z}(\mathcal{L}) g-g=\left[\psi_{R}(\mathcal{L}) m_{R}^{z}(\mathcal{L})-1\right] e^{-\frac{1}{t} \mathcal{L}} e^{-s \mathcal{L}} f
$$

Thus,

$$
\begin{aligned}
\left|m_{R}^{z}(\mathcal{L}) g(x)-g(x)\right| & =\left|\left[\psi_{R}(\mathcal{L}) m_{R}^{z}(\mathcal{L})-1\right] e^{-\frac{1}{t} \mathcal{L}} e^{-s \mathcal{L}} f(x)\right| \\
& \leq\left\|\left[\psi_{R}(\mathcal{L}) m_{R}^{z}(\mathcal{L})-1\right] e^{-\frac{1}{t} \mathcal{L}} h_{s}(x, \cdot)\right\|_{2}\|f\|_{2} \\
& \leq \sup _{\lambda>0}\left[\psi_{R}(\lambda) m_{R}^{z}(\lambda)-1\right] e^{-\frac{1}{t}(1-\gamma(\lambda))}\left\|h_{s}(x, \cdot)\right\|_{2}\|f\|_{2},
\end{aligned}
$$

where the last inequality follows from the spectral theorem. Since

$$
\sup _{\lambda>0}\left[\psi_{R}(\lambda) m_{R}^{z}(\lambda)-1\right] e^{-\frac{1}{t}(1-\gamma(\lambda))} \rightarrow 0, \text { as } R \rightarrow \infty,
$$

we obtain

$$
\left|m_{R}^{z}(\mathcal{L}) g(x)-g(x)\right| \rightarrow 0, \text { as } R \rightarrow \infty,
$$

Step 3: Theorem 1 holds on the whole class $L^{p}(\mathbb{T}), 1 \leq p \leq 2$.

As in [1], it suffices to combine Steps 1 and 2, and well-known measure theoretic arguments, see for example [13, Theorem 2.1.14], and the proof of Theorem 1 is complete. Note that since the measure on homogeneous trees is discrete, almost everywhere convergence reduces to everywhere convergence.

\section{References}

[1] G. Alexopoulos, N. Lohoué, Riesz means on Lie groups and Riemannian manifolds of nonnegative curvature, Bull. Soc. Math. France, (122), no. 2, (1994), 209-223.

[2] J. Bertrand, P. Bertrand, J.P. Ovarlez, The Mellin transform, in: The Transforms and Applications Handbook, ed. A.D. Poularikas, CRC Press Inc., 1999, Chapter 18.

[3] M. Christ, Weak type (1; 1) bounds for rough operators, Ann. of Math., 128, no. 2 (1988), 19-42.

[4] M. Christ, C. Sogge, Weak type $L^{1}$ convergence of eigenfunction expansions for pseudodifferential operators, Invent. Math., 94 (1988), 421-453.

[5] J.L. Clerc, Sommes de Riesz et multiplicateurs sur un groupe de Lie compact, Ann. Inst. Fourier, 24 (1974), 149-172.

[6] M. Cowling, Harmonic analysis on semigroups, Ann. of Math., 117 (1983), 267-283.

[7] M. Cowling, S. Meda, A.G. Setti, Estimates for functions of the Laplace operator on homogeneous trees, Trans. Am. Math. Soc. 352, no. 9, (2000), 4271-4293.

[8] A.J. Eddine, Schrödinger equations on homogeneous trees, J. Lie Theory, 23 (2013), 779-794.

[9] A. Figà-Talamanca, C. Nebbia: Harmonic analysis and representation theory for groups acting on homogeneous trees, London Math. Soc. Lect. Notes Ser. 162, Cambridge University Press, 1991.

[10] A. Fotiadis, A.G. Georgiadis, Riesz means on graphs and discrete groups, Potential Anal. 38 (2013), 21-30.

[11] A. Fotiadis, E. Papageorgiou, Riesz means on symmetric spaces, J. Math. Anal. Appl., 499, no.1, (2021).

[12] S. Giulini, G. Mauceri, Almost everywhere convergence of Riesz means on certain noncompact symmetric spaces, Ann. di Mat. Pura ed Appl. (1991), 357-369.

[13] L. Grafakos, (2004). Classical and modern Fourier analysis. New Jersey: Pearson Education.

[14] M. Marias, $L^{p}$-boundedness of oscillating spectral multipliers on Riemannian manifolds, Ann. Math. Blaise Pascal, 10 (2003), 133-160.

[15] C. Nebbia, Groups of isometries of a tree and the Kunze-Stein phenomenon, Pacific J. Math., 133 (1988), 141-149.

[16] A. Seeger, Endpoint estimates for multiplier transformations on compact manifolds, Indiana Univ. Math. J., 40, no. 2, (1991), 471-533.

[17] A. Setti, $L^{p}$ and operator norm estimates for the complex time heat operator on homogeneous trees, Trans. Amer. Math. Soc., 350, no. 2 (1988), 743--768.

[18] C. Sogge, On the convergence of Riesz means on compact manifolds, Ann. of Math., 126, no. 3, (1987), 439-447.

[19] E.M. Stein, Localization and summability of multiple Fourier series, Acta Math., 100 (1958), 93-147.

[20] E.M. Stein, Topics in Harmonic Analysis Related to the Littlewood-Paley Theory, (AM-63), Volume 63, Princeton University Press, Princeton, 1971.

[21] E.M. Stein, C. Weiss, Introduction to Fourier Analysis on Euclidean Spaces, Princeton University Press, Princeton, 1971.

[22] J. Tits, Sur le groupe des automorphismes d'un arbre, Mémoires dédiés à Georges de Rham, Springer Verlag, Berlin, 1970, $188-211$ 